\title{
1 Characteristics and outcomes of hospitalized adult COVID-19 patients in Georgia
}

2 Tengiz Tsertsvadze, MD, PhD,${ }^{1,2}$ Marina Ezugbaia, MD, ${ }^{1}$ Marina Endeladze, MD,${ }^{1}$ Levani

3 Ratiani MD, PhD, ${ }^{3}$ Neli Javakhishvili MD, ${ }^{4}$ Lika Mumladze $\mathrm{MD},{ }^{5}$ Manana Khotchava MD,

4 PhD, ${ }^{6}$ Maiko Janashia MD, ${ }^{7}$ Diana Zviadadze MD,${ }^{8}$ Levan Gopodze MD,${ }^{9}$ Alex Gokhelashvili

$5 \quad \mathrm{MD},{ }^{10}$ Revaz Metchurchtlishvili, MD, ${ }^{1}$ Akaki Abutidze, MD, MPH, PhD ${ }^{1,2}$ Nikoloz

6 Chkhartishvili, MD, MS, $\mathrm{PhD}^{1,11}$

$8 \quad{ }^{1}$ Infectious Diseases, AIDS and Clinical Immunology Research Center, Tbilisi, Georgia

$9 \quad{ }^{2}$ Ivane Javakhishvili Tbilisi State University, Tbilisi, Georgia

$10 \quad{ }^{3}$ First University Clinic, Tbilisi, Georgia

$11{ }^{4}$ Giorgi Abramishvili Military Hospital, Gori, Georgia

12 St. King Mirian and Queen Nana Mtskheta Regional Medical Center, Georgia

$13 \quad{ }^{6}$ Children's Infectious Diseases Hospital, Tbilisi, Georgia

$14 \quad{ }^{7}$ University Clinic Rukhi, Georgia

$15 \quad{ }^{8}$ LJ Clinic Kutaisi, Georgia

$16{ }^{9}$ Central Republican Hospital, Tbilisi, Georgia

$17 \quad{ }^{10}$ Medalpha Clinic, Batumi, Georgia

$18{ }^{11}$ Caucasus International University, Tbilisi, Georgia

20 Corresponding author: Tengiz Tsertsvadze, MD, PhD 


\section{Abstract}

25 Objective: Describe presenting characteristics of hospitalized patients and explore factors

26 associated with in-hospital mortality during the first wave of pandemic in Georgia.

27 Methods: This retrospective study included 582 adult patients admitted to 9 dedicated COVID-

2819 hospitals as of July 30, 2020 (72\% of all hospitalizations). Data were abstracted from medical

29 charts. Factors associated with mortality were evaluated in multivariable Poisson regression

30 analysis.

31 Results: Among 582 adults included in this analysis $14.9 \%$ were $65+$ years old, $49.1 \%$ were

32 women, $59.3 \%$ had uni- or bi-lateral lung involvement on chest computed tomography, $27.1 \%$

33 had any co-morbidity, $13.2 \%$ patients had lymphopenia, $4.1 \%$ had neutophilosis, $4.8 \%$ had low

34 platelet count, $37.6 \%$ had d-dimer levels of $>0.5 \mathrm{mcg} / \mathrm{l}$. Overall mortality was $2.1 \%(12 / 582)$.

35 After excluding mild infections, mortality among patients with moderate-to-critical disease was

$363.0 \%$ (12/399), while among patients with severe-to-critical disease mortality was $12.7 \%(8 / 63)$.

37 Baseline characteristics associated with increased risk of mortality in multivariate regression

38 analysis included: age $\geq 65$ years (RR: $10.38,95 \%$ CI: $1.30-82.75$ ), presence of any chronic co-

39 morbidity (RR: 20.71, 95\% CI: 1.58-270.99), lymphopenia (RR: 4.76, 95\% CI: 1.52-14.93),

40 neutrophilosis (RR: 7.22, 95\% CI: 1.27-41.12), low platelet count (RR: $6.92,95 \%$ CI: 1.18 -

41 40.54), elevated d-dimer (RR: 4.45, 95\% CI: 1.48-13.35), elevated AST (RR: 6.33, 95\% CI:

$42 \quad 1.18-33.98)$.

43 Conclusion: In-hospital mortality during the first wave of pandemic in Georgia was low. We

44 identified several risk factors (older age, co-morbidities and laboratory abnormalities) associated

45 with poor outcome that should provide guidance for planning health sector response as pandemic

46 continues to evolve. 


\section{Introduction}

50 Ongoing pandemic of novel coronavirus disease 2019 (COVID-19) showed differential impact

51 on mortality across the European continent, with cumulative mortality ranging from 1 to over

52100 death per 100,000 population (1). Georgia is a small Eastern European country that rapidly

53 responded to the pandemic threat by implementing effective public health and clinical measures

54 achieving one of the lowest mortality rates of 1.25 per 100,000 population as of October 5,2020

55 (1). The care pathway model included testing with real-time reverse transcriptase polymerase

56 chain reaction (RT-PCR) of suspected cases (contacts of confirmed cases, persons with

57 symptoms suggestive of COVID-19) with rapid hospitalization of all confirmed cases regardless

58 of disease severity. Sixteen "fever" centres have been established for initial triage and RT-PCR

59 diagnostics of symptomatic persons, while in-patient care was provided in 12 dedicated COVID-

6019 hospitals. The objective of this study was to describe presenting characteristics of hospitalized

61 patients and explore factors associated with in-hospital mortality during the first wave of

62 pandemic.

63

64

65

66

67

68 


\section{Methods}

72 This retrospective study included unselected consecutive patients with RT-PCR confirmed

73 COVID-19 admitted to 9 designated COVID-19 hospitals across the country between February

7426 and July 30, 2020, for whom complete outcome data was known (discharged alive or died in

75 hospital).

As of July 30, 2020, 1160 persons with confirmed COVID-19 have been hospitalized in Georgia,

78 among them outcome was available for 952 patients of whom 935 (98.2\%) were discharged alive

79 and 17 (1.8\%) died. For present analysis complete outcome was available for 678 hospitalized

80 persons (582 adults and 96 children) of whom 666 (98.2\%) were discharged alive and $12(1.8 \%)$

81 died. All lethal outcomes were registered among adults and therefore analysis was limited to

82 persons aged $\geq 18$ years.

84 Data were extracted from medical charts, including demographic characteristics, symptoms at

85 presentation, vital signs, laboratory assays, presence of chronic co-morbid conditions,

86 complications during hospital stay, admission to intensive care unit (ICU), need for oxygen

87 support, treatment strategy and outcome of hospitalization. Treatment is provided in accordance

88 with the national protocols, which is the "living guideline" regularly updated with emerging

89 evidence. Confirmed cases were classified into mild, moderate, severe and critical disease.

90

91

92 


\section{Results}

95 Among 582 adults included in this analysis the median age was 46 (IQR: $34-58$ ) years, $14.9 \%$

96 were $65+$ years old, $49.1 \%$ were women. At the time of hospitalization $13.1 \%$ patients had no

97 symptoms, 59.3\% had uni- or bi-lateral lung involvement on chest CT and $27.1 \%$ patients had

98 any chronic co-morbid condition (cardiovascular disease, diabetes, cancer, chronic obstructive

99 pulmonary disease, chronic kidney disease, chronic liver disease). With regard to baseline

100 laboratory values $13.2 \%$ patients had lymphopenia, $4.1 \%$ had neutophilosis, $4.8 \%$

101 thrombocytopenia, $37.6 \%$ had d-dimer levels of $>0.5 \mathrm{mcg} / \mathrm{l}, 14.8 \%$ patients had AST levels $>40$

102 IU/l and 14.3\% had creatinine levels of $>100 \mathrm{mcmol} / \mathrm{l}$. Based on baseline characteristics 183

$103(31.4 \%)$ patients were classified as having mild disease, $336(57.7 \%)$ - moderate disease, 53

$104(9.1 \%)$ - severe disease and $10(1.7 \%)$-critical disease.

105

106 Over the median length of stay of 20 (IQR: 16-24) days 12 adult died with mortality of $2.1 \%$.

107 Baseline characteristics associated with increased risk of mortality in multivariate regression

108 analysis included: age $\geq 65$ years (RR: $10.38,95 \%$ CI: $1.30-82.75$ ), presence of any chronic co-

109 morbidity (RR: 20.71, 95\% CI: 1.58-270.99), lymphocyte count <1 X 109\%/ (RR: 4.76, 95\% CI:

$1101.52-14.93)$, neutrophil count $>8 \times 10^{9} / 1(\mathrm{RR}: 7.22,95 \%$ CI: $1.27-41.12)$, platelet count $<150 \mathrm{X}$

$11110 \%$ / (RR: 6.92, 95\% CI: 1.18-40.54), d-dimer >0.5 mcg/ml (RR: 4.45, 95\% CI: $1.48-13.35$ ),

112 AST >40 IU/l (RR: 6.33, 95\% CI: 1.18-33.98) (Table 1).

113

114 Mortality rates by severity of disease at presentation were: $0.0 \%(0 / 183)$ for mild disease, $1.2 \%$

115 (4/336) for moderate, 3.8\% (2/53) for severe disease and 60\% (6/10) for critical disease (Table 
medRxiv preprint doi: https://doi.org/10.1101/2020.10.23.20218255; this version posted October 27, 2020. The copyright holder for this preprint (which was not certified by peer review) is the author/funder, who has granted medRxiv a license to display the preprint in perpetuity.

It is made available under a CC-BY-NC-ND 4.0 International license .

116 2). After excluding mild infections, mortality among patients with moderate-to-critical disease

117 was 3.0\% (12/399), while among patients with severe-to-critical disease mortality was $12.7 \%$

118 (8/63).

119

120 A total of $91(15.6 \%)$ patients developed complications during hospital stay, including $25(4.3 \%)$

121 patients developing multiple complications resulting in 11 deaths. Spectrum of complications

122 included $43(7.4 \%)$ new episodes of pneumonia, 19 (3.3\%) acute respiratory distress syndrome, 6

$123(1.0 \%)$ cardiovascular events (3 acute coronary disease, 2 ischemic strokes, 1 haemorrhagic

124 stroke), $19(3.3 \%)$ sepsis/septic shock, 6 (1.0\%) acute liver injury, 10 (1.7\%) acute kidney injury,

$12519(3.3 \%)$ cytokine storm (Table 2). Forty-one (7.0\%) patients required admission to ICU

126 resulting in 12 deaths (29.3\% mortality) and $44(7.6 \%)$ patients required oxygen support

127 resulting in 12 deaths $(27.3 \%$ mortality) (Table 2$)$.

128

129 Overall $282(48.5 \%)$ patient received standard of care treatment only, $291(50.0 \%)$ received

130 hydroxycholorquine and 9 (4.8\%) - lopinavir/ritonavir. In addition, $338(58.1 \%)$ patients

131 received antibiotics, $52(8.9 \%)$ - corticosteroids, $2(0.3 \%)$ - tocilizumab, $3(0.5 \%)$ -

132 plasmapheresis, $3(0.5 \%)$ blood purification with cytosrobents and $2(0.3 \%)$ transfusion of

133 convalescent plasma.

134

135

136

137

138 


\section{Discussion}

140

141 In this cohort of consecutive hospitalized adult patients with laboratory confirmed COVID-19 we

142 found low in-hospital mortality of $2.1 \%$ among total population, $3 \%$ - among patients with at

143 least moderate disease and $12.7 \%$ among patient with at least severe disease at presentation.

144 Overall mortality shown in our study is lower than $18.88 \%$ reported in recent meta-analysis, even

145 if we limit our analysis to patients with severe/critical disease (2). However, the difference needs

146 to be interpreted with caution in light of varying criteria for hospitalization and rationing of

147 resources in overburdened healthcare systems (3). Reported rates of mortality among patients

148 admitted to ICU or requiring ventilation ranges between $23 \%$ and $53 \%$ (4-7), with our study

149 showing mortality of $29.3 \%$.

151 Similar to other studies older age, presence of chronic comorbidities and laboratory

152 abnormalities (leukopenia, neutrophilosis, thromocytopeia and increase in d-dimer) have been

153 significantly associated with mortality (8-11). Interestingly, our data did not show gender-based

154 differences neither in univariate nor multivariate analysis., while reports from other countries

155 universally indicate increased risk of death among men (12).

157 Predicting outcomes of COVID_19 disease has been a subject of intensive research (13). This is

158 particularly important for Georgia as the country moved from hospitalize all approach to

159 outpatient care of persons with mild disease because of significant increase in diagnosis since

160 September 2020 (1). Defining criteria for hospitalization is crucial for appropriate utilization of

161 healthcare resources without compromising lives. In our study baseline clinical characteristics, 
162 such as fever, abnormalities in respiratory rate, oxygen saturation and chest CT imaging showed

163 significant association with mortality only in univariate analysis. As an example, 4 patients in

164 our cohort, initially classified to have moderate disease, had significant deterioration of their

165 condition leading to death. All these 4 patients had pre-existing cardiovascular disease, 3 had

166 elevated d-dimer along with lymphopenia - combination of factors that had been shown to

167 predict progression of disease (14). This suggests that initial clinical presentation may not be

168 sufficient for predicting outcome, it is rather complex issue that requires combinations of several

169 determinants, including laboratory parameters.

170

171 Our analysis included 72\% of COVID-19 hospitalizations occurring in Georgia during the first

172 wave of pandemic thus ensuring representativeness of national data. Limitations include small

173 sample size and small number of events that affected statistical power. Analysis was limited to

174 laboratory parameters included in basic package of care, while other biomarkers known to be

175 associated with outcome such as albumin, IL-6, ferritin (15), were available only for small subset

176 of patients.

177

178 In summary, in-hospital mortality during the first wave of pandemic in Georgia was low. Our

179 study characterized risk factors (older age, co-morbidities and laboratory abnormalities)

180 associated with poor outcome that should provide guidance for planning health sector response

181 as pandemic continues to evolve.

182 


\section{References}

187 1. European Centre for Disease Prevention and Control. Situation dashboard - COVI-19

188 cases in Europe and worldwide. Stockholm: ECDC; 2020 [cited 2020 October 5]; Available

189 from: https://qap.ecdc.europa.eu/public/extensions/COVID-19/COVID-19.html.

190 2. Noor FM, Islam MM. Prevalence and Associated Risk Factors of Mortality Among

191 COVID-19 Patients: A Meta-Analysis. J Community Health. 2020.

192 3. Emanuel EJ, Persad G, Upshur R, Thome B, Parker M, Glickman A, et al. Fair Allocation

193 of Scarce Medical Resources in the Time of Covid-19. N Engl J Med. 2020 May

$194 \quad 21 ; 382(21): 2049-55$.

195 4. Argenziano MG, Bruce SL, Slater CL, Tiao JR, Baldwin MR, Barr RG, et al.

196 Characterization and clinical course of 1000 patients with coronavirus disease 2019 in New

197 York: retrospective case series. BMJ. 2020:m1996.

198 5. Docherty AB, Harrison EM, Green CA, Hardwick HE, Pius R, Norman L, et al. Features

199 of $20 \square 133$ UK patients in hospital with covid-19 using the ISARIC WHO Clinical

200 Characterisation Protocol: prospective observational cohort study. BMJ. 2020:m1985.

201 6. Grasselli G, Zangrillo A, Zanella A, Antonelli M, Cabrini L, Castelli A, et al. Baseline

202 Characteristics and Outcomes of 1591 Patients Infected With SARS-CoV-2 Admitted to ICUs of

203 the Lombardy Region, Italy. Jama. 2020 Apr 28;323(16):1574-81.

204 7. Karagiannidis C, Mostert C, Hentschker C, Voshaar T, Malzahn J, Schillinger G, et al.

205 Case characteristics, resource use, and outcomes of $10 \square 021$ patients with COVID-19 admitted to

206920 German hospitals: an observational study. Lancet Resp Med. 2020;8(9):853-62. 
medRxiv preprint doi: https://doi.org/10.1101/2020.10.23.20218255; this version posted October 27, 2020. The copyright holder for this preprint (which was not certified by peer review) is the author/funder, who has granted medRxiv a license to display the preprint in perpetuity. It is made available under a CC-BY-NC-ND 4.0 International license .

207 8. Bonanad C, García-Blas S, Tarazona-Santabalbina F, Sanchis J, Bertomeu-González V,

208 Fácila L, et al. The Effect of Age on Mortality in Patients With COVID-19: A Meta-Analysis

209 With 611,583 Subjects. J Am Med Dir Assoc. 2020;21(7):915-8.

$2109 . \quad$ Harrison SL, Fazio-Eynullayeva E, Lane DA, Underhill P, Lip GYH. Comorbidities

211 associated with mortality in 31,461 adults with COVID-19 in the United States: A federated

212 electronic medical record analysis. PLoS Med. 2020 Sep;17(9):e1003321.

213 10. Martins-Filho PR, Tavares CSS, Santos VS. Factors associated with mortality in patients

214 with COVID-19. A quantitative evidence synthesis of clinical and laboratory data. European

215 Journal of Internal Medicine. 2020;76:97-9.

216 11. Zhang L, Yan X, Fan Q, Liu H, Liu X, Liu Z, et al. D $\square$ dimer levels on admission to

217 predict in $\square$ hospital mortality in patients with Covid $\square$ 19. J Thromb Haemost. 2020;18(6):13242189.

219 12. Haitao T, Vermunt JV, Abeykoon J, Ghamrawi R, Gunaratne M, Jayachandran M, et al.

220 COVID-19 and Sex Differences: Mechanisms and Biomarkers. Mayo Clin Proc. 2020

221 Oct;95(10):2189-203.

222 13. Gupta RK, Marks M, Samuels THA, Luintel A, Rampling T, Chowdhury H, et al.

223 Systematic evaluation and external validation of 22 prognostic models among hospitalised adults

224 with COVID-19: An observational cohort study. Eur Respir J. 2020 Sep 25.

225 14. Feng Z, Yu Q, Yao S, Luo L, Zhou W, Mao X, et al. Early prediction of disease

226 progression in COVID-19 pneumonia patients with chest CT and clinical characteristics. Nature

227 Communications. 2020;11(1).

228 15. Henry BM, de Oliveira MHS, Benoit S, Plebani M, Lippi G. Hematologic, biochemical

229 and immune biomarker abnormalities associated with severe illness and mortality in coronavirus 
medRxiv preprint doi: https://doi.org/10.1101/2020.10.23.20218255; this version posted October 27, 2020. The copyright holder for this preprint (which was not certified by peer review) is the author/funder, who has granted medRxiv a license to display the preprint in perpetuity.

230 disease 2019 (COVID-19): a meta-analysis. Clinical Chemistry and Laboratory Medicine

231 (CCLM). 2020;58(7):1021-8.

232

233

234

235

236

237

238

239

240

241

242

243

244

245

246

247

248

249

250

251

252 


\begin{tabular}{|c|c|c|c|c|c|c|}
\hline & \multirow[b]{2}{*}{ Total, n (\%) } & \multirow[b]{2}{*}{ Died, $n(\%)$} & \multicolumn{2}{|c|}{ Univariate analysis } & \multicolumn{2}{|c|}{ Multivariate analysis } \\
\hline & & & RR $(95 \%$ CI $)$ & p value & RR $(95 \%$ CI $)$ & p value \\
\hline \multicolumn{7}{|l|}{ Age categories } \\
\hline $18-65$ & $495(85.1)$ & $2(0.4)$ & 1 & & 1 & \\
\hline $65+$ & $87(14.9)$ & $10(11.5)$ & $28.45(6.34-127.63)$ & $<0.0001$ & $10.38(1.30-82.75)$ & 0.03 \\
\hline \multicolumn{7}{|l|}{ Gender } \\
\hline Female & $286(49.1)$ & $7(2.4)$ & 1 & & 1 & \\
\hline Male & $296(50.9)$ & $5(1.7)$ & $0.69(0.22-2.15)$ & 0.52 & $1.82(0.47-7.08)$ & 0.38 \\
\hline \multicolumn{7}{|l|}{ Symptoms other then fever } \\
\hline Asymptomatic & $76(13.1)$ & $1(1.3)$ & 1 & & 1 & \\
\hline Symptomatic & $506(97.8)$ & $11(2.2)$ & $1.65(0.21-12.62)$ & 0.63 & $0.17(0.01-4.23)$ & 0.28 \\
\hline \multicolumn{7}{|l|}{ Temperature } \\
\hline$<37$ & $252(43.3)$ & $4(1.6)$ & 1 & & 1 & \\
\hline $37-<38$ & $271(46.6)$ & $4(1.5)$ & $0.93(0.24-3.68)$ & 0.92 & $0.27(0.06-1.16)$ & 0.08 \\
\hline $38+$ & $59(10.1)$ & $4(6.8)$ & $4.27(1.10-16.59)$ & 0.04 & $0.25(0.06-1.17)$ & 0.08 \\
\hline \multicolumn{7}{|l|}{ Respiratory rate } \\
\hline$<24$ & $520(89.3)$ & $4(0.8)$ & 1 & & 1 & \\
\hline $24+$ & $62(10.7)$ & $8(12.9)$ & $16.78(5.20-54.10)$ & $<0.0001$ & $1.90(0.25-14.58)$ & 0.53 \\
\hline \multicolumn{7}{|l|}{ Oxygen saturation } \\
\hline$\geq 94$ & $530(91.1)$ & $6(1.1)$ & 1 & & 1 & \\
\hline$<94$ & $52(8.9)$ & $6(11.5)$ & $10.19(3.41-30.47)$ & $<0.0001$ & $1.69(0.37-7.75)$ & 0.50 \\
\hline \multicolumn{7}{|l|}{ Chest CT } \\
\hline No lung involvement & $237(40.7)$ & $1(0.4)$ & 1 & & 1 & \\
\hline Uni- or Bi-lateral involvement & $345(59.3)$ & $11(3.19)$ & $7.56(0.98-58.14)$ & 0.05 & $1.51(0.09-24.62)$ & 0.77 \\
\hline \multicolumn{7}{|l|}{ Comorbidities } \\
\hline No comorbidities & $424(72.9)$ & $1(0.2)$ & 1 & & 1 & \\
\hline Any comorbidity & $158(27.1)$ & $11(7.0)$ & $29.52(3.84-226.78)$ & 0.001 & $21.15(1.60-280.56)$ & 0.02 \\
\hline
\end{tabular}




\begin{tabular}{|c|c|c|c|c|c|c|}
\hline \multicolumn{7}{|l|}{ BMI } \\
\hline$<25$ & $238(40.9)$ & $5(2.1)$ & 1 & & 1 & \\
\hline $25-30$ & $283(48.6)$ & $4(1.4)$ & $0.67(0.18-2.48)$ & 0.55 & $0.19(0.02-1.48)$ & 0.11 \\
\hline$>30$ & $61(10.5)$ & $3(4.9)$ & $2.34(0.58-9.53)$ & 0.23 & $1.50(0.29-7.88)$ & 0.63 \\
\hline \multicolumn{7}{|l|}{ Hemoglobin } \\
\hline$\geq 12 \mathrm{~g} / \mathrm{dl}$ & $521(89.5)$ & $8(1.5)$ & 1 & & 1 & \\
\hline$<12 \mathrm{~g} / \mathrm{dl}$ & $61(10.5)$ & $4(6.6)$ & $4.27(1.32-13.77)$ & 0.02 & $1.67(0.54-5.14)$ & 0.37 \\
\hline \multicolumn{7}{|c|}{ White blood count } \\
\hline$\leq 11 \times 10^{9} / 1$ & $564(96.9)$ & $9(1.6)$ & 1 & & 1 & \\
\hline$>11 \times 10^{9} / 1$ & $18(3.1)$ & $3(16.7)$ & $10.44(3.09-35.36)$ & 0.002 & $3.29(0.31-35.26)$ & 0.33 \\
\hline \multicolumn{7}{|c|}{ Lymphocyte count } \\
\hline$\geq 1 \times 10^{9} / 1$ & $505(86.8)$ & $6(1.2)$ & 1 & & 1 & \\
\hline$<1 \times 10^{9} / 1$ & $77(13.2)$ & $6(7.8)$ & $6.56(2.17-19.82)$ & 0.0009 & $4.74(1.48-15.10)$ & 0.008 \\
\hline \multicolumn{7}{|c|}{ Neutrophil count } \\
\hline$\leq 8 \times 10^{9} / 1$ & $558(95.9)$ & $9(1.6)$ & 1 & & 1 & \\
\hline$>8 \times 10^{9} / 1$ & $24(4.1)$ & $3(12.5)$ & $7.75(2.24-26.81)$ & 0.001 & $7.27(1.26-41.89)$ & 0.03 \\
\hline \multicolumn{7}{|l|}{ Platelet count } \\
\hline$\geq 150 \times 10^{9} / 1$ & $553(95.2)$ & $10(1.8)$ & 1 & & 1 & \\
\hline$<150 \times 10^{9} / 1$ & $28(4.8)$ & $2(7.1)$ & $3.94(0.91-17.18)$ & 0.07 & $6.99(1.20-40.61)$ & 0.03 \\
\hline \multicolumn{7}{|l|}{ D-dimer } \\
\hline$\leq 0.5 \mathrm{mcg} / \mathrm{ml}$ & $363(62.4)$ & $2(0.6)$ & 1 & & 1 & \\
\hline$>0.5 \mathrm{mcg} / \mathrm{ml}$ & $219(37.6)$ & $10(4.6)$ & $8.29(1.83-37.48)$ & 0.006 & $4.47(1.51-13.24)$ & 0.007 \\
\hline \multicolumn{7}{|c|}{$\mathrm{C}$ reactive protein } \\
\hline$\leq 5 \mathrm{mg} / 1$ & $214(36.8)$ & $2(0.9)$ & 1 & & 1 & \\
\hline$>5 \mathrm{mg} / \mathrm{l}$ & $368(63.2)$ & $10(2.7)$ & $2.91(0.64-13.15)$ & 0.17 & $1.26(0.17-9.51)$ & 0.82 \\
\hline \multicolumn{7}{|l|}{ ALT } \\
\hline$\leq 40 \mathrm{IU} / 1$ & $468(80.4)$ & $9(1.9)$ & 1 & & 1 & \\
\hline$>40 \mathrm{IU} / 1$ & $114(19.6)$ & $3(2.6)$ & $1.37(0.38-4.97)$ & 0.63 & $4.54(0.77-26.93)$ & 0.10 \\
\hline
\end{tabular}




\begin{tabular}{|c|c|c|c|c|c|c|}
\hline \multicolumn{7}{|l|}{ AST } \\
\hline$\leq 40 \mathrm{IU} / 1$ & $496(85.2)$ & $7(1.4)$ & 1 & & 1 & \\
\hline$>40 \mathrm{IU} / 1$ & $86(14.8)$ & $5(5.8)$ & $4.12(1.34-12.68)$ & 0.01 & $6.34(1.18-34.13)$ & 0.03 \\
\hline \multicolumn{7}{|l|}{ Creatinine } \\
\hline$\leq 100 \mathrm{mcmol} / 1$ & $499(85.7)$ & $8(1.6)$ & 1 & & 1 & \\
\hline$>100 \mathrm{mcmol} / \mathrm{l}$ & $83(14.3)$ & $4(4.8)$ & $3.01(0.93-9.76)$ & 0.07 & $1.88(0.56-6.31)$ & 0.31 \\
\hline
\end{tabular}


255 Table 2. Mortality by severity of disease and complications during hospital stay $(\mathbf{n}=\mathbf{5 8 2})$

\begin{tabular}{|c|c|c|c|c|}
\hline & $\begin{array}{c}\text { Total } \\
(\mathrm{n}=\mathbf{5 8 2})\end{array}$ & $\begin{array}{c}\text { Discharged alive } \\
(n=570)\end{array}$ & $\begin{array}{c}\text { Died } \\
(n=12)\end{array}$ & p value \\
\hline \multicolumn{5}{|c|}{ Severity of disease at baseline, n (\%) } \\
\hline Mild & $183(31.4)$ & $183(100.0)$ & $0(0.0)$ & 0.0007 \\
\hline Moderate & $336(57.7)$ & $332(98.8)$ & $4(1.2)$ & \\
\hline Severe & $53(9.1)$ & $51(96.2)$ & $2(3.8)$ & \\
\hline Critical & $10(1.7)$ & $4(40.0)$ & $6(60.0)$ & \\
\hline \multicolumn{5}{|c|}{ Complications during hospital stay, n (\%) } \\
\hline None & $491(84.4)$ & $491(100.0)$ & $0(0.0)$ & $<0.0001$ \\
\hline 1 complication & $66(11.3)$ & $65(98.5)$ & $1(1.5)$ & \\
\hline$>1$ complication & $25(4.3)$ & $14(56.0)$ & $11(44.0)$ & \\
\hline \multicolumn{5}{|l|}{ Pneumonia, n (\%) } \\
\hline No & $539(92.6)$ & $528(98.0)$ & $11(2.0)$ & 0.61 \\
\hline Yes & $43(7.4)$ & $42(97.7)$ & $1(2.3)$ & \\
\hline \multicolumn{5}{|l|}{ ARDS, n (\%) } \\
\hline No & $554(95.2)$ & $552(99.6)$ & $2(0.4)$ & $<0.0001$ \\
\hline Yes & $28(4.8)$ & $18(64.3)$ & $10(35.7)$ & \\
\hline \multicolumn{5}{|c|}{ Cardiovascular events, n (\%) } \\
\hline None & $576(99.0)$ & $568(98.6)$ & $8(1.4)$ & $<0.0001$ \\
\hline Acute coronary disease & $3(0.5)$ & $0(0.0)$ & $3(100.0)$ & \\
\hline Ischemic stroke & $2(0.3)$ & $2(100.0)$ & $0(0.0)$ & \\
\hline Hemorrhagic stroke & $1(0.2)$ & $0(0.0)$ & $1(100.0)$ & \\
\hline \multicolumn{5}{|l|}{ Sepsis/septic shock, n (\%) } \\
\hline No & $563(96.7)$ & $562(99.8)$ & $1(0.2)$ & $<0.0001$ \\
\hline Yes & $19(3.3)$ & $8(42.1)$ & $11(57.9)$ & \\
\hline \multicolumn{5}{|l|}{ Acute liver injury, n (\%) } \\
\hline No & $576(99.0)$ & $565(98.1)$ & $11(1.9)$ & 0.11 \\
\hline Yes & $6(1.0)$ & $5(83.3)$ & $1(16.7)$ & \\
\hline \multicolumn{5}{|c|}{ Acute kidney injury, n (\%) } \\
\hline No & $572(98.3)$ & $565(98.8)$ & $1(1.2)$ & $<0.0001$ \\
\hline Yes & $10(1.7)$ & $5(50.0)$ & $1(50.0)$ & \\
\hline \multicolumn{5}{|l|}{ Cytokine storm, n (\%) } \\
\hline No & $563(96.7)$ & $554(98.4)$ & $9(1.6)$ & 0.005 \\
\hline Yes & $19(3.3)$ & $16(84.2)$ & $3(15.8)$ & \\
\hline \multicolumn{5}{|l|}{ Admission to ICU, n (\%) } \\
\hline No & $541(93.0)$ & $541(100.0)$ & $0(0.0)$ & $<0.0001$ \\
\hline Yes & $41(7.0)$ & $29(70.7)$ & $12(29.3)$ & \\
\hline \multicolumn{5}{|l|}{ Oxygen support, n (\%) } \\
\hline No oxygen support & $538(92.4)$ & $538(100.0)$ & $0(0.0)$ & $<0.0001$ \\
\hline Oxygen support, n (\%) & $44(7.6)$ & $32(72.7)$ & $12(27.3)$ & \\
\hline
\end{tabular}


medRxiv preprint doi: https://doi.org/10.1101/2020.10.23.20218255; this version posted October 27, 2020. The copyright holder for this preprint (which was not certified by peer review) is the author/funder, who has granted medRxiv a license to display the preprint in perpetuity. 